\title{
船-
}

\section{Relación entre los estilos de Liderazgo Transformacional y Laissez-faire y el Síndrome de Quemarse por el Trabajo en profesores de educación secundaria}

\author{
JORGE Jesús LÓPEZ-VÍlChez \\ UnidAd de INVESTIGACIÓN PSICOSOCIAL DE LA CONDUCTA \\ Organizacional (UNIPSICO). UnIVERSITAT de VALĖNCIA \\ jorlovil@alumni.uv.es \\ Ester Grau-Alberola \\ UnidAD de INVESTIGACIÓN PSICOSOCIAL DE LA CondUCtA \\ ORGaNIZACIONAL (UNIPSICO) \\ UnIVERSIDAD INTERNACIONAL DE LA RIOJA (UNIR) \\ ester.grau@unir.net \\ Pedro R. Gill-Monte \\ Unidad de InVESTIGACIÓN Psicosocial de LA Conducta \\ OrganizaCIONAL (UNIPSICO). UnIVERSITAT de VAlĖNCIA \\ Pedro.Gil-Monte@uv.es \\ Hugo Figueiredo-FerRaz \\ UnidAD de INVESTIGACIÓN PSICOSOCIAL DE LA CONDUCTA \\ ORGANIZACIONAL (UNIPSICO) \\ UnIVERSIDAD INTERNACIONAL DE VALENCIA (VIU) \\ hugo.figueiredo@campusviu.es
}

\section{Resumen:}

Introducción: los objetivos del estudio fueron los siguientes: (1) analizar la influencia de los estilos de Liderazgo Transformacional (LT) y Laissez-faire (LLF) sobre el 
Síndrome de Quemarse por el Trabajo (SQT); (2) evaluar la influencia de las dimensiones de LT (Carisma o Influencia idealizada, Inspiración, Estimulación intelectual y Consideración individualizada) sobre las dimensiones de SQT Perfil 1 (llusión por el trabajo, Desgaste psíquico e Indolencia).

Material y métodos: la muestra estuvo formada por 211 profesores portugueses de educación secundaria $(28.9 \%$ hombres y $71.1 \%$ mujeres). LT (Carisma o Influencia idealizada, Inspiración, Estimulación intelectual y Consideración individualizada) y LLF fueron evaluados mediante una versión reducida de la Escala Multifactor Leadership Questionnaire (MLQ). Por su parte, el Perfil 1 de SQT (Ilusión por el trabajo, Desgaste psíquico e Indolencia) se evaluó mediante el Cuestionario para la Evaluación del Síndrome de Quemarse por el Trabajo (CESQT) en su versión para profesionales del sector educativo.

Resultados: el análisis realizado permitió obtener los siguientes resultados: (1) influencia significativa y negativa entre LT y SQT globales; (2) influencia significativa y positiva entre LT e llusión por el trabajo; (3) influencia significativa y negativa entre Inspiración y SQT global; (4) influencia significativa y positiva entre Consideración individualizada e llusión por el trabajo.

Discusión: en base a los resultados obtenidos se recomienda el impulso en los centros docentes de educación secundaria de políticas de recursos humanos orientadas a la formación de líderes transformacionales, como factor determinante en la mejora de los niveles de salud ocupacional, eficacia y rendimiento organizacional.

Palabras clave: Liderazgo transformacional, liderazgo laissez-faire, riesgos psicosociales, síndrome de quemarse por el trabajo, salud ocupacional.

\section{Relationship between Transformational and Laissez-faire leadership styles and Burnout in secondary school teachers}

\section{Abstract:}

Introduction: the aims of this study were as follows: (1) to analyze the influence of Transformational Leadership (TL) and Laissez-faire Leadership (LFL) on Occupational Burnout Syndrome (OBS); (2) to evaluate the influence of the dimensions of TL (Charisma or Idealized Influence, Inspirational Motivation, Intellectual Stimulation and Individualized Consideration) on the dimensions of OBS Profile 1 (Enthusiasm toward the job, Psychological exhaustion and Indolence). 
Method: the sample consisted of 211 Portuguese secondary school teachers (28.9\% male and $71.1 \%$ female). Transformational Leadership (Charisma or Idealized Influence, Inspirational Motivation, Intellectual Stimulation and Individualized Consideration) and Laissez-faire Leadership were assessed using a specific set of items from the Multifactor Leadership Questionnaire (MLQ). In order to assess the levels in Occupational Burnout Syndrome Profile 1 (Enthusiasm towards the job, Psychological Exhaustion and Indolence) we employed the "Spanish Burnout Inventory" (SBI) in its version for education professionals.

Results: the analysis brought up the following results: (1) significant and negative influence between TL and global OBS; (2) significant and positive influence between TL and Enthusiasm towards the job; (3) significant and negative influence between Inspirational Motivation and global OBS; (4) significant and positive influence between Individualized Consideration and Enthusiasm towards the job.

Discussion: based on the results obtained, it is recommended that human resources policies aimed to train transformational leaders inside secondary educational institutions, as an essential component to improve the levels of occupational health, efficiency and organizational performance.

Key words: Transformational leadership, laissez-faire leadership, psychosocial risks, burnout, occupational health. 



\section{Relación entre los estilos de}

Liderazgo Transformacional

y Laissez-faire y el Síndrome

de Quemarse por el Trabajo

en profesores de educación secundaria

Jorge Jesús

López-Vílchez

Ester

Grau-Alberola

Pedro R.

Gil-Monte

Hugo

Figueiredo-Ferraz

https://doi.org/10.26754/ojs_ais/ais.2019393239

Recibido: 02/10/2017

Aceptado: 12/04/2018

\section{INTRODUCCIÓN}

Condiciones de trabajo y riesgos psicosociales en el sector educativo

Durante las últimas décadas hemos asistido a múltiples y rápidos cambios que han modificado y reconfigurado el mercado laboral tal como lo conocíamos, a través del planteamiento de nuevas formas de organización del trabajo y división de las tareas. Las condiciones laborales derivadas de estos contextos han traído consigo nuevos riesgos y daños potenciales para la salud de los trabajadores (Unda et al., 2008), siendo el sector de la educación uno de los más afectados por dichos cambios (Liu y Onwuegbuzie, 2012; Wiegel et al., 2016). El estudio que planteamos en este artículo se circunscribe en particular a la etapa educativa de secundaria.

El número de exigencias y demandas en los colegios e institutos no ha dejado de aumentar en los últimos años. Los cambios pedagógicos (marcados por el rápido avance tecnológico y las frecuentes modificaciones legislativas en educación) y sociales (definidos por las distintas concepciones hacia la figura 
del profesor y el papel que debe desempeñar) han afectado de forma sustancial a los profesionales de este sector, generando una serie de tensiones constantes y diarias en el trabajo.

Este contexto ha creado una situación definida como "malestar en el profesorado" (Martínez, 2015: 1), la cual se explica en base a una serie de agentes causales, entre los cuales podemos destacar los siguientes: multiplicidad de roles del profesor, imagen idealizada del mismo, recursos escasos en los centros educativos, falta de flexibilidad organizacional, violencia en la escuela y dificultad para adaptarse a las innovaciones (Figueiredo-Ferraz et al., 2013).

En este sentido, una de las causas más importantes de malestar que afecta a los profesores en la actualidad es el estrés laboral, el cual supone un problema de gran repercusión, especialmente dentro de este sector (Gastaldi et al., 2014).

Desde las instituciones europeas se ha destacado la gran preocupación existente en los últimos años dentro del ámbito educativo por las cuestiones relacionadas con el estrés laboral. Según la Encuesta Europea de Empresas sobre Riesgos Nuevos y Emergentes (ESENER) (Agencia Europea para la Seguridad y Salud en el Trabajo, EU-OSHA, 2010), este sector fue uno de los que mostró una mayor inquietud por esta materia (concretamente más del $80 \%$ de profesores), únicamente por detrás de los profesionales del sector sanidad y trabajo social (donde las cifras alcanzan el 90\%).

Por otra parte, un primer avance de la última versión de esta encuesta (ESENER-2) (Agencia Europea para la Seguridad y la Salud en el Trabajo, EU-OSHA, 2015), señala que países como España y particularmente Portugal cuentan con un porcentaje de empresas con planes de acción para prevenir el estrés laboral inferior a la media de la Unión Europea.

Los procesos de estrés originados en el entorno laboral repercuten de forma muy relevante en la salud del profesorado, ya que se asocian frecuentemente al incremento de los accidentes laborales (Pérez, 2010), absentismo (Moriana y Herruzo, 2004) y a problemas de salud de carácter grave (Doménech y Gómez, 2010).

\section{Síndrome de Quemarse por el Trabajo}

El Síndrome de Quemarse por el Trabajo (en adelante SQT) se define como una respuesta al estrés laboral crónico característico 
de los profesionales que trabajan hacia otras personas (Gil-Monte, 2005). Ha sido objeto de gran interés desde el ámbito de la Psicología de la Salud Ocupacional en los últimos años, ya que constituye uno de los daños laborales de índole psicosocial más relevantes en la sociedad actual (Salanova y Llorens, 2008).

En este sentido, las investigaciones recientes hacen referencia a la presencia de una serie de riesgos psicosociales en el entorno docente que pueden actuar como desencadenantes de los procesos de SQT: sobrecarga laboral (Antoniou et al., 2006), falta de autonomía y participación (Carlotto y Palazzo, 2006), ambigüedad de rol (Kokkinos, 2007), conflictos interpersonales con alumnos, padres y compañeros de trabajo (Betoret, 2006; Skaalvik y Skaalvik, 2007) y conflicto trabajo-familia (Camargo et al., 2015).

Factores como los anteriores configuran un entorno de trabajo caracterizado por un creciente número de demandas cada vez más intensas, exigentes e incluso contradictorias que el docente, aun realizando su máximo esfuerzo dentro y fuera del horario de trabajo habitual, se ve imposibilitado para cumplir. Esta situación de excesiva carga de trabajo simultánea puede desencadenar en último término síntomas de estrés crónico y manifestarse a través de importantes niveles de despersonalización y agotamiento emocional (De la Cruz y Urdiales, 1996), alterando no sólo la eficiencia profesional del individuo, sino también su salud física y psicológica.

La evaluación del SQT en este estudio se realizó mediante el Cuestionario para la Evaluación del Síndrome de Quemarse por el Trabajo (en adelante CESQT) (Gil-Monte, 2011), que valora un total de cuatro dimensiones que componen el síndrome:

a) Ilusión por el trabajo: hace referencia a la aspiración del sujeto por alcanzar las metas laborales, que le suponen a su vez una fuente de satisfacción o placer personal.

b) Desgaste psíquico: agotamiento físico o emocional que sufre el individuo, fruto del trato diario en el trabajo con personas que presentan o causan problemas.

c) Indolencia: desarrollo de actitudes negativas de cinismo e indiferencia hacia los clientes de la organización, tales como estudiantes o familiares (Richmond et al., 2009). 
d) Culpa: sentimientos de culpa en el sujeto debidos a su actitud negativa en el trabajo, en especial hacia las personas con las que trabaja.

A su vez, el modelo teórico en el que se basa el CESQT permite diferenciar entre dos perfiles de individuos en función del desarrollo o no de sentimientos de Culpa:

Perfil 1: caracteriza a todos aquellos sujetos que sufren en un primer momento un deterioro cognitivo y afectivo (con bajas puntuaciones en la dimensión llusión por el trabajo y altas puntuaciones en Desgaste psíquico), como respuesta a la exposición a fuentes de estrés laboral crónico. Posteriormente los individuos desarrollan actitudes negativas dirigidas a las personas que atienden en su trabajo (niveles elevados de Indolencia).

Perfil 2: constituye frecuentemente un problema de mayor entidad que caracteriza a los individuos más deteriorados por el SQT. En esta situación los sujetos, además de los síntomas anteriores, también manifiestan niveles elevados de sentimientos de Culpa. La finalidad de este estudio no consistió en realizar un análisis exhaustivo del desarrollo del SQT y sus niveles de prevalencia, por ello los análisis se realizaron considerando únicamente el Perfil 1.

Los procesos de SQT generan a su vez una serie de consecuencias: por una parte deterioran y merman la calidad del servicio ofrecido por las instituciones educativas y por la otra, también conducen a alteraciones en la salud de profesores y profesoras, a través de menores niveles de motivación por su trabajo, absentismo, depresión e insomnio (Mercado y Gil-Monte, 2012).

Respecto a los niveles de prevalencia del síndrome en el sector educativo a nivel internacional, los estudios realizados en los últimos años destacan importantes porcentajes: entre el $11 \%$ y el 15\% en Portugal y España (Figueiredo-Ferraz et al., 2009; Longas et al., 2012), $21.4 \%$ en Uruguay (Ratto et al., 2015) y $35.5 \%$ en México (Unda, 2010).

\section{Liderazgo Transformacional y Laissez-faire}

Dentro del contexto laboral definido en los apartados anteriores, las técnicas de liderazgo organizacional suponen una herramienta esencial, permitiendo una gestión más adecuada de 
los recursos humanos que forman parte de las instituciones educativas y facilitando la función docente mediante el apoyo y el asesoramiento adecuados (Mendoza y García, 2013).

El colectivo de profesores y profesoras se encuentra expuesto a factores de riesgo psicosocial derivados de las intensas demandas de interacción social que caracterizan a su puesto: impartición de clases en el aula a grupos de alumnos, atención particularizada en su horario de tutorías, reuniones periódicas con padres y sesiones de trabajo conjunto con el resto de compañeros y compañeras a través de claustros semanales. Este contexto situacional hace necesario un liderazgo adecuado en los centros escolares que faciliten la función del docente.

No obstante, para que dicha ayuda resulte adecuada y permita satisfacer a su vez las expectativas de los empleados debe desarrollarse de forma eficaz, en este sentido una de las competencias básicas consiste en orientar a los empleados sin llegar a coartar su propia autonomía. Un líder implicado en la resolución de problemas, dispuesto a proporcionar apoyo a sus seguidores e interesado en su satisfacción representa una ventaja competitiva diferencial frente a otras organizaciones (Rome, 2000), ya que permite potenciar las capacidades de cada uno de sus trabajadores, optimizando la eficacia y eficiencia de su capital humano.

En el estudio propuesto se van a analizar dos estilos de liderazgo opuestos entre sí: Laissez-faire y Transformacional (en adelante LLF y LT).

En primer lugar el LLF se define por varios autores como un estilo que implica per se la ausencia o falta de liderazgo. Caracteriza a aquellos líderes poco comprometidos e implicados en su empresa, con tendencia a evitar la toma de decisiones y la supervisión responsable del grupo que tienen a su mando. Entregan el poder y la autoridad a sus seguidores intencionadamente, aunque sin perder completamente el control, liberándose de cualquier responsabilidad (Pacsi et al., 2014).

Otras de sus características son las siguientes: falta de definición de objetivos y metas colectivas con claridad, ineficacia en la resolución de conflictos (debida a su actitud de evitación permanente de los mismos) y ausencia de dirección y apoyo al grupo del que forman parte, situación que deriva en un amplio número de casos en una disminución de la eficacia y eficiencia globales 
en el centro de trabajo y en una menor satisfacción e implicación de sus empleados.

En el extremo opuesto se encuentra el LT: supone un estilo de dirección muy distinto al anterior, caracteriza a aquellos líderes que desean motivar a sus empleados no sólo para que le sigan, sino también para que cuestionen el orden y las prácticas establecidas en su empresa (Bass y Avolio, 1990) con el objetivo de generar cambios que permitan una mejora en el rendimiento organizacional. Sus principales características son las siguientes:

a) Carisma o Influencia idealizada: permite la proyección de una visión de futuro optimista sobre la organización como equipo, buscando el bienestar común orientado al logro de las metas colectivas más allá de los intereses o beneficios individuales. El líder consigue captar la atención de sus empleados gracias los comportamientos y actitudes singulares con las que transmite su mensaje, unidas al propio atractivo personal que despierta entre ellos de forma natural.

b) Inspiración (o Motivación inspiracional): consiste en la capacidad de motivar a los seguidores transmitiéndoles la creencia de que podrán alcanzar los objetivos planteados y superar las dificultades que puedan surgir. De este modo les estimula y fortalece su autoestima para que sean capaces de hacer frente a situaciones más complejas.

c) Estimulación intelectual: los líderes transformacionales fomentan la creatividad e innovación de sus empleados, evitando sancionar públicamente sus aportaciones. De este modo los trabajadores se sienten más seguros y motivados para proponer nuevas alternativas de actuación, lo cual permite superar el status quo imperante en el entorno de trabajo.

d) Consideración individualizada: el líder apoya a sus seguidores, buscando su desarrollo profesional y personal. Trata de implicarse con cada empleado atendiendo sus necesidades de forma particularizada y escuchando activamente sus preocupaciones, lo cual facilita la percepción de un liderazgo realmente operativo e implicado en la solución de problemas.

El estilo transformacional persigue satisfacer en los empleados las necesidades de alto orden, relacionadas con el reconocimiento, el sentido de pertenencia y la autorrealización, haciéndoles conscientes de la importancia de sus aportaciones en el logro 
de la misión colectiva del centro y proporcionándoles la confianza suficiente para que sean capaces de asumir por sí mismos nuevas responsabilidades (Mittal, 2015).

Las investigaciones recientes han confirmado la existencia de relaciones entre los estilos de LT y LLF y las dimensiones del SQT, entre ellas Ilusión por el trabajo e Indolencia (Hetland et al., 2007; Kanste et al., 2007). Dichas investigaciones constituyen el punto de partida de este estudio.

\section{Objetivos e hipótesis}

Los objetivos propuestos fueron los siguientes: (1) analizar la influencia de los estilos de LT y LLF sobre el SQT en la muestra objeto de estudio y (2) evaluar la influencia de cada una de las dimensiones constituyentes del LT (Carisma o Influencia idealizada, Inspiración, Estimulación intelectual y Consideración individualizada) sobre las dimensiones que forman parte del Perfil 1 del SQT (llusión por el trabajo, Desgaste psíquico e Indolencia).

Para ello se plantearon las siguientes hipótesis:

Hipótesis 1: los resultados obtenidos confirmarán la influencia negativa y significativa del estilo de LT sobre el SQT (H1a). Del mismo modo, se confirmará la influencia positiva y significativa entre el estilo de LLF y el SQT (H1b). Todas las variables incluidas en esta hipótesis preliminar serán consideradas a nivel global.

\section{Figura 1}

\section{Modelo gráfico}

(H1a)

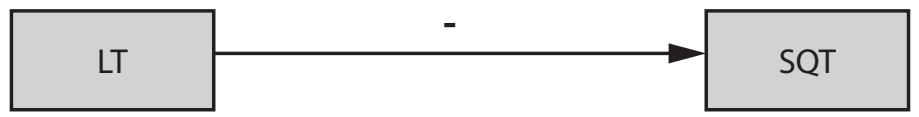

(H1b)

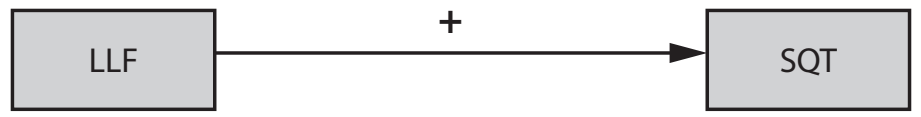

Hipótesis 2: Las dimensiones que componen el LT influirán de forma negativa y significativa en el SQT globalmente considerado (H2a, H2b, H2c y H2d). 
Figura 2

Modelo gráfico

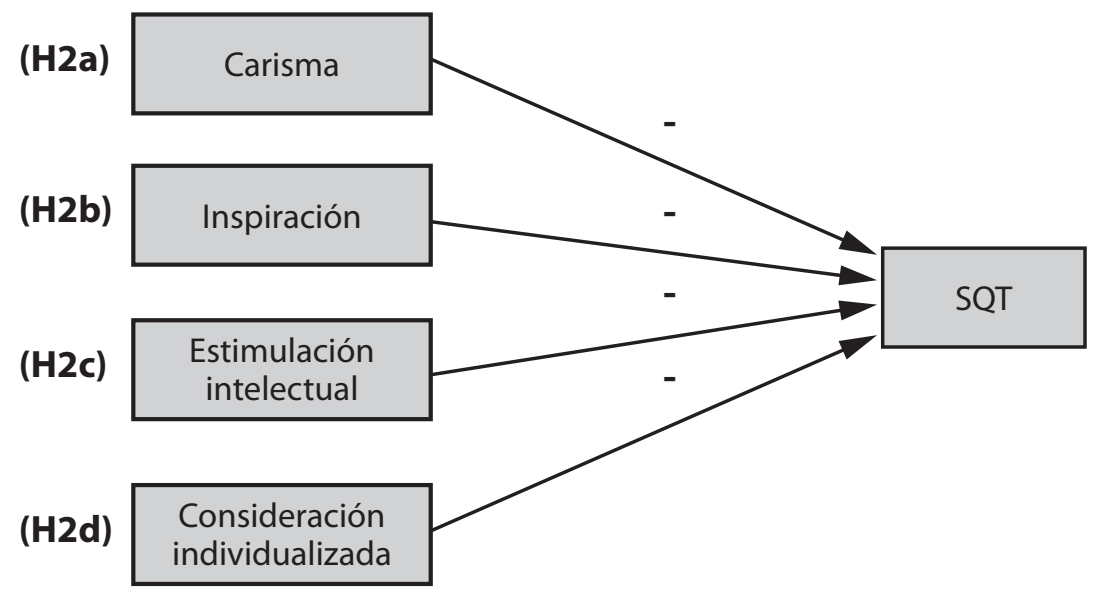

Hipótesis 3: Las dimensiones componentes del LT influirán significativamente y en la dirección esperada en las dimensiones de SQT:

(H3a) Influencia significativa y positiva de las dimensiones Carisma o Influencia idealizada, Inspiración, Estimulación intelectual y Consideración individualizada sobre la dimensión llusión por el trabajo. 
Figura 3

Modelo gráfico

(H3a)

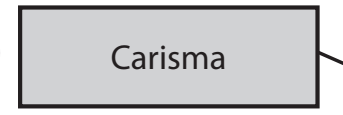

(H3a)

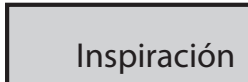

Inspiración

(H3a)

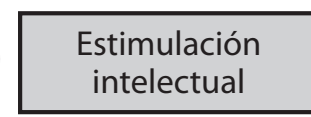

(H3a)

Consideración individualizada

(H3b) Influencia significativa y negativa de las dimensiones Carisma o Influencia idealizada, Inspiración, Estimulación intelectual y Consideración individualizada sobre la dimensión Desgaste psíquico.

\section{Figura 4}

\section{Modelo gráfico}

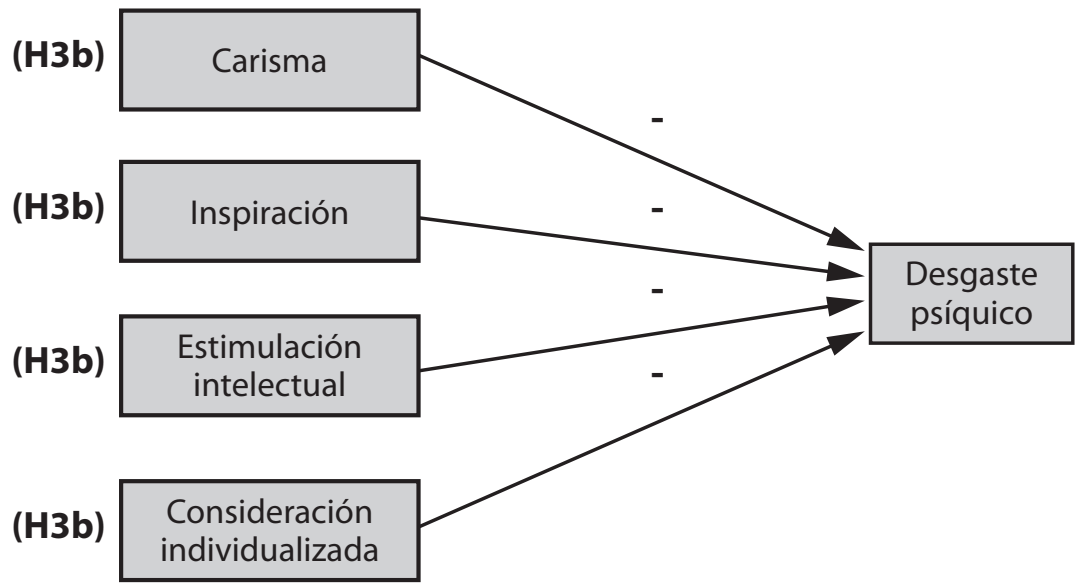


(H3c) Influencia significativa y negativa de las dimensiones Carisma o Influencia idealizada, Inspiración, Estimulación intelectual y Consideración individualizada sobre la dimensión Indolencia.

\section{Figura 5}

\section{Modelo gráfico}

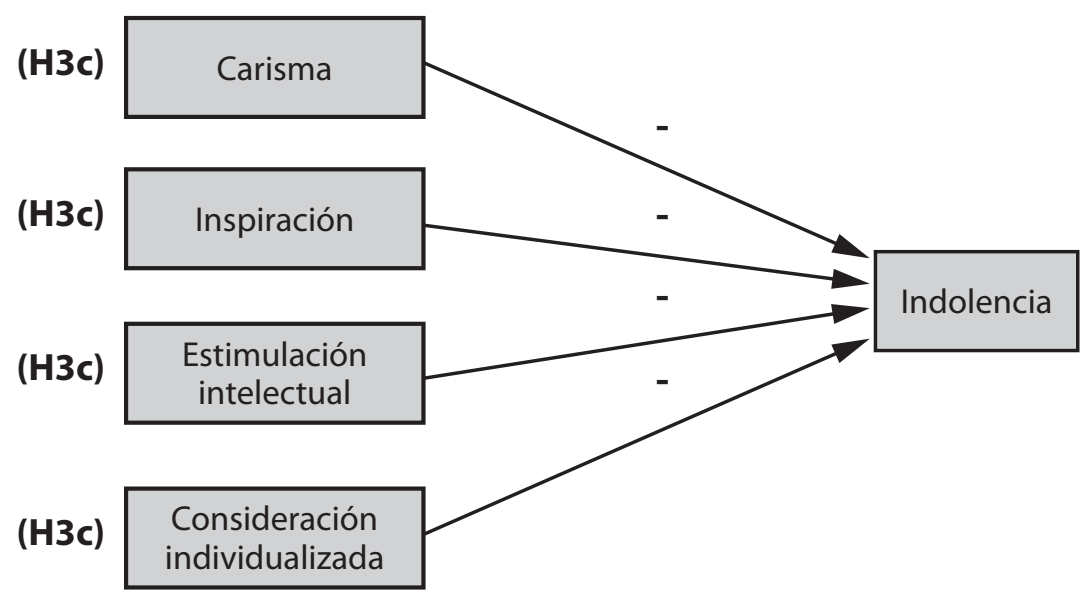

\section{MATERIAL Y MÉTODOS}

\section{Participantes}

La muestra objeto de estudio estuvo compuesta por un total de 211 profesores de educación secundaria procedentes de cinco colegios distintos de la Región Norte de Portugal, de los cuales 61 (28.90\%) fueron hombres y 150 (71.10\%) mujeres. Dicha composición concuerda con la información correspondiente al año 2015 proporcionada por la Organización de las Naciones Unidas para la Educación, la Ciencia y la Cultura (UNESCO, 2017), en la cual se indica que el porcentaje de docentes mujeres en educación secundaria en Portugal fue del $70 \%$. La media de edad de la muestra fue de 41.23 años $(\mathrm{dt}=9.24$, $\max .=68$, min. =23). Por tipo de contrato, $132(62.60 \%)$ trabajadores tenían contrato fijo, 17 (8.10\%) pertenecían al cuadro de zona pedagógica, y $24(11.40 \%)$ tenían un contrato temporal. A esta pregunta no contestaron $38(18 \%)$ de los participantes en el estudio. 
Respecto a la antigüedad en el puesto, la media fue de 17.48 $(\mathrm{dt}=9.74$, max. $=37$, min. $=0)$, mientras que la antigüedad en la organización obtuvo una media de $12.81(\mathrm{dt}=10.23$, $\max .=36$, min. =0). Por último, en lo referente al estado civil, 167 (79.10\%) tenían una relación estable en el momento en que respondieron al cuestionario.

\section{Diseño}

El estudio planteado responde a los criterios de un diseño transversal. Mediante el mismo, se procederá a realizar el análisis de las relaciones de influencia entre las variables expuestas en el apartado introductorio, junto con sus respectivas dimensiones componentes.

\section{Procedimiento}

Los participantes fueron seleccionados mediante la técnica de muestreo no aleatorio. La recogida de datos se realizó en diversos centros escolares ubicados en la zona norte de Portugal. Para ello, se envió previamente un fax dirigido a los Consejos Ejecutivos de cada uno de los colegios, en el cual se solicitaba autorización para proceder a la entrega de los cuestionarios. Una vez recibida su respuesta afirmativa, se entregaron los cuestionarios a los presidentes de cada uno de los Consejos Ejecutivos de cada colegio, los cuales se encargaron a su vez de realizar la distribución en los distintos departamentos, donde fueron rellenados por los participantes voluntariamente. Los cuestionarios fueron distribuidos en los centros junto con un sobre de respuesta para hacerlos llegar de nuevo a los investigadores. El ratio de respuesta de la muestra objeto de estudio fue del $84.4 \%$.

\section{Instrumentos de medida}

Para llevar a cabo la evaluación de los estilos de LT y LLF se utilizó una versión reducida de la Escala Multifactor Leadership Questionnaire (MLQ) (Bass y Avolio, 1990), formada por un total de 15 ítems que miden las cuatro dimensiones de LT: (a) Carisma o Influencia idealizada (3 ítems, $\alpha=.92$ ); (b) Inspiración (3 ítems, $\alpha=.86$ ); (c) Estimulación intelectual (3 ítems, $\alpha=.72$ ); 
(d) Consideración individualizada ( 3 ítems, $\alpha=.85$ ) (total LT: 12 ítems, $\alpha=.95$ ); $y$ el estilo LLF ( 3 ítems, $\alpha=.65$ ).

A título ilustrativo, la escala anterior incluye ítems tales como los siguientes: LT: (a) Carisma o Influencia idealizada ("mi jefe/a de estudios, o directorla en su defecto, cree y me transmite la importancia de nuestra misión"); (b) Inspiración ("mi jefe/a de estudios, o directorla en su defecto, desarrolla formas de motivarnos"); (c) Estimulación intelectual ("mi jefe/a de estudios, o directorla en su defecto, me transmite la importancia de trabajar mucho y bien"); (d) Consideración individualizada ("mi jefe/a de estudios, o directorla en su defecto, da consejos a quienes los necesitan"); y LLF ("mi jefe/a de estudios, o director/a en su defecto evita involucrarse en mi trabajo"). Los ítems se evaluaron con una escala de frecuencia de cinco grados (0 "Nunca" a 4 "Muy frecuentemente: todos los días").

Por su parte, tal como se indicó en el apartado de introducción, el SQT fue evaluado mediante el cuestionario CESQT (GilMonte, 2011; Gil-Monte y Figueiredo-Ferraz, 2013). En este estudio se empleó su versión para docentes (Gil-Monte et al., 2010; Rabasa et al., 2016). Dicho cuestionario está formado por un total de 20 ítems ( $\alpha=.87)$ que se distribuyen en cuatro dimensiones: (a) Ilusión por el trabajo (5 ítems, $\alpha=.88$ ); (b) Desgaste psíquico (4 ítems, $\alpha=.81$ ); (c) Indolencia (6 ítems, $\alpha=.75$ ); y (d) Culpa (5 ítems, $\alpha=.75$ ). Siguiendo el criterio indicado en el apartado de introducción, la dimensión Culpa finalmente no se incluyó en los análisis al formar parte del Perfil 2 del SQT.

Las escalas que forman cada una de las dimensiones analizadas del CESQT incluyeron ítems tales como los siguientes: (a) Ilusión por el trabajo ("me siento ilusionado/a por mi trabajo); (b) Desgaste psíquico ("me siento desgastado/a emocionalmente); (c) Indolencia ("pienso que trato con indiferencia a algunos alumnos/as"). Los ítems se evaluaron con una escala de frecuencia de cinco grados (0 "Nunca" a 4 "Muy frecuentemente: todos los días").

Por último, el cálculo de las variables globales LT, LLF y SQT se llevó a cabo mediante el mismo procedimiento en todas ellas: consistió en el cálculo de la puntuación promedio que otorgaron los profesores y profesoras a los distintos ítems que componían a su vez cada variable. Siguiendo el criterio mantenido desde el 
apartado introductorio, no se incluyeron en este cálculo los ítems correspondientes a la variable Culpa, por ser característica del perfil 2 de SQT.

\section{Análisis de datos}

Para comprobar las hipótesis planteadas se realizaron análisis descriptivos, de correlación y regresión empleando para ello el paquete estadístico SPSS versión 21 y AMOS versión 23.

\section{RESULTADOS}

\section{Estadísticos descriptivos y correlaciones de LT/LLF y SQT globales y dimensionales}

En primer lugar se obtuvo la matriz de correlaciones entre las variables LT, LLF y SQT consideradas a nivel global, junto con sus respectivas dimensiones componentes (Tabla 1). El análisis de correlación exploratorio permite confirmar la existencia de varias relaciones significativas entre el LT y el SQT no sólo a nivel global, sino también entre sus dimensiones. Así, por ejemplo, puede observarse en dicha tabla cómo el LT global correlacionó de forma negativa y significativa con el SQT global $(r=-.16 ; p=.02)$ y de forma positiva y significativa con la dimensión llusión $(r=.28 ; p=.00)$. Sin embargo, el estilo de LLF no obtuvo correlaciones significativas con SQT global $(r=.04 ; p=.54)$ ni con ninguna de sus dimensiones componentes: Ilusión $(r=-.01 ; p=.86)$, Desgaste psíquico $(r=.06 ; p=.38)$ e Indolencia $(r=.03 ; p=.70)$. Por su parte, el SQT global obtuvo correlaciones positivas y significativas con las dimensiones Inspiración ( $r=-.18 ; p=.01)$, Consideración individualizada $(r=-.15 ; p=.03)$ y Carisma $(r=-.17 ; p=.01)$. 


\section{Tabla 1}

\section{Estadísticos descriptivos y matriz de correlaciones entre las variables objeto de estudio}

\begin{tabular}{|l|c|c|c|c|c|c|c|c|c|c|c|c|c|}
\hline & Media & $\mathbf{d t}$ & Rango & $\mathbf{1}$ & $\mathbf{2}$ & $\mathbf{3}$ & $\mathbf{4}$ & $\mathbf{5}$ & $\mathbf{6}$ & $\mathbf{7}$ & $\mathbf{8}$ & $\mathbf{9}$ & $\mathbf{1 0}$ \\
\hline 1. llusión & 2.88 & 0.70 & $0-4$ & & & & & & & & & & \\
\hline 2. Desgaste & 1.85 & 0.83 & $0-4$ & $-.43^{\star \star}$ & & & & & & & & & \\
\hline 3. Indolencia & 1.20 & 0.62 & $0-4$ & $-.36^{\star \star}$ & $.43^{\star \star}$ & & & & & & & & \\
\hline 4. SQT global & 1.34 & 0.55 & $0-4$ & $-.77^{\star \star}$ & $.78^{\star \star}$ & $.78^{\star \star}$ & & & & & & & \\
\hline 5. Estim. intel. & 1.78 & 0.83 & $0-4$ & $.22^{\star \star}$ & -.04 & .05 & -.08 & & & & & & \\
\hline 6. Inspiración & 1.78 & 0.92 & $0-4$ & $.27^{\star \star}$ & -.13 & -.02 & $-.18^{\star \star}$ & $.78^{\star \star}$ & & & & & \\
\hline 7. Consid. ind. & 2.06 & 0.96 & $0-4$ & $.28^{\star \star}$ & -.09 & .02 & $-.15^{\star}$ & $.70^{\star \star}$ & $.87^{\star \star}$ & & & & \\
\hline 8. Carisma & 1.91 & 1.02 & $0-4$ & $.26^{\star \star}$ & -.13 & -.02 & $-.17^{\star}$ & $.73^{\star \star}$ & $.88^{\star \star}$ & $.86^{\star \star}$ & & & \\
\hline 9. LLF global & 2.05 & 0.82 & $0-4$ & -.01 & .06 & .03 & .04 & $-.20^{\star \star}$ & $-.22^{\star \star}$ & $-.19^{\star \star}$ & $-.20^{\star \star}$ & & \\
\hline 10. LT global & 1.88 & 0.87 & $0-4$ & $.28^{\star \star}$ & -.11 & .00 & $-.16^{\star}$ & $.86^{\star \star}$ & $.95^{\star \star}$ & $.93^{\star \star}$ & $.94^{\star \star}$ & $-.22^{\star \star}$ & \\
\hline
\end{tabular}

$$
{ }^{*} p<.05,{ }^{* *} p<.01
$$

\section{Relación de los estilos de liderazgo (LT /LLF) globales con SQT global}

A continuación se realizaron una serie de análisis de regresión mediante el procedimiento de escalonamiento paso a paso (stepwise). Con el objetivo de maximizar el grado de ajuste en los modelos de regresión planteados, empleando para ello el mínimo número posible de variables, se decidió optar por incluir en los análisis las variables Sexo y Edad como variables de control. Los resultados obtenidos fueron los siguientes:

En primer lugar se comprobó la influencia de los estilos de liderazgo descritos sobre el SQT (Tabla 2) (Figura 6). En este caso todas las variables introducidas fueron consideradas a nivel global. Se obtuvo una influencia estadísticamente significativa y negativa entre LT y SQT ( $\beta=-.15, p=.02)$, confirmando la primera de las hipótesis planteadas (H1a). Sin embargo, no fue posible confirmar la siguiente hipótesis $(\mathrm{H} 1 \mathrm{~b})$, ya que no se encontraron influencias significativas entre el estilo de LLF y el $\operatorname{SQT}(\beta=.03, p=.70)$. 


\section{Tabla 2}

\section{Resultados análisis LT y LLF globales con SQT global (Regresión Stepwise)}

\begin{tabular}{|c|c|c|c|c|}
\hline & & SQT global & & \\
\hline & Pasos & $\beta$ & Cambio R $^{2}$ & $\mathrm{~F}$ \\
\hline \multirow{2}{*}{1} & Sexo & $.15^{\star}$ & .04 & $4.20^{\star}$ \\
\cline { 2 - 5 } & Edad & $.14^{*}$ & & \\
\hline 2 & LT global & $-.15^{\star}$ & .05 & $4.54^{\star \star}$ \\
\hline
\end{tabular}

${ }^{* *} p \leq .01,{ }^{*} p \leq .05$

\section{Figura 6}

Resultados Hipótesis 1, modelo gráfico

(H1a)

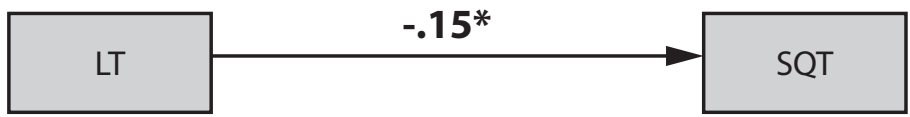

(H1b)

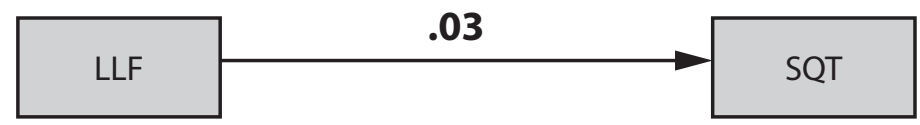

${ }^{* *} p \leq .01,{ }^{*} p \leq .05$

Relación de los estilos de liderazgo (LT/ LLF) globales con las dimensiones de SQT

Posteriormente se analizó la influencia ejercida por el LT y LLF (considerados a nivel global) sobre cada una de las dimensiones componentes del SQT (Tabla 3). En primer lugar, los análisis reflejaron una influencia estadísticamente significativa del LT sobre la dimensión llusión $(\beta=.27, p=.00)$, sin embargo esta relación no se dio con LLF ( $\beta=.04, p=.60)$. Por su parte, no se hallaron relaciones de influencia significativa de $\operatorname{LT}(\beta=-.09, p=.16)$ ni de $\operatorname{LLF}(\beta=.08, p=.29)$ sobre la dimensión Desgaste. Por último, LT no influyó significativamente sobre la dimensión Indolencia $(\beta=.01, p=.90)$, al igual que ocurrió con LLF $(\beta=.03, p=.62)$. 


\section{Tabla 3}

\section{Resultados análisis LT y LLF globales con dimensiones SQT (Regresión Stepwise)}

\begin{tabular}{|c|c|c|c|c|}
\hline & & Ilusión & & \\
\hline & Pasos & $\beta$ & $\mathrm{R}^{2}$ & $\mathrm{~F}$ \\
\hline \multirow{2}{*}{1} & Sexo & -.12 & \multirow{2}{*}{.01} & $2.51^{\star}$ \\
\cline { 2 - 3 } & Edad & -.08 & & \\
\hline 2 & LT global & $.27^{\star \star}$ & .08 & $7.51^{\star \star}$ \\
\hline
\end{tabular}

${ }^{* *} p \leq .01,{ }^{*} p \leq .05$

\section{Relación de las dimensiones de LT con SQT global}

Mediante el siguiente análisis, se comprobó la influencia de cada una de las dimensiones componentes del LT en el SQT globalmente considerado (Tabla 4) (Figura 7). En este caso la dimensión Inspiración fue la única que influyó en el desarrollo del SQT (influencia estadísticamente significativa y negativa) $(\beta=-.17, p=.01)$, mientras que para el resto de dimensiones de LT no se obtuvo ninguna influencia significativa sobre el SQT: Estimulación intelectual $(\beta=.15, p=.15)$, Consideración individualizada $(\beta=.04, p=.78)$ y Carisma $(\beta=-.05, p=.73)$ lo cual nos lleva al rechazo de las hipótesis $\mathrm{H} 2 \mathrm{a}, \mathrm{H} 2 \mathrm{c}$ y H2d pero nos permite confirmar la hipótesis $\mathrm{H} 2 \mathrm{~b}$.

\section{Tabla 4}

Resultados análisis dimensiones LT con SQT global (Regresión Stepwise)

\begin{tabular}{|c|c|c|c|c|}
\hline & & SQT global & & \\
\hline \multirow{2}{*}{1} & Pasos & $\beta$ & $\mathrm{R}^{2}$ & $\mathrm{~F}$ \\
\hline & Sexo & $.14^{\star}$ & \multirow{2}{*}{.04} & $4.20^{\star}$ \\
\cline { 2 - 5 } & Edad & $.15^{\star}$ & & \\
\hline 2 & Inspiración & $-.17^{\star *}$ & .05 & $5.09^{\star *}$ \\
\hline
\end{tabular}

$$
{ }^{* *} p \leq .01,{ }^{*} p \leq .05
$$




\section{Figura 7}

Resultados Hipótesis 2, modelo gráfico

(H2a)

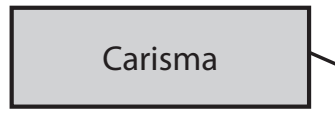

(H2b)
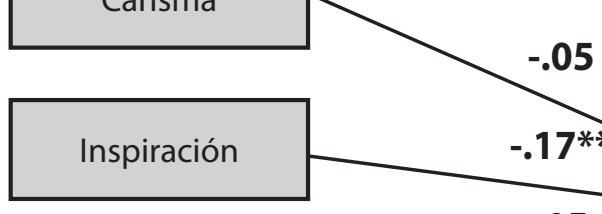

(H2c)

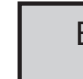

Estimulación intelectual

(H2d)

Consideración individualizada

${ }^{* *} p \leq .01,{ }^{*} p \leq .05$

\section{Relación de las dimensiones LT con las dimensiones SQT}

Por último, se analizó la influencia de cada una de las dimensiones componentes del estilo transformacional sobre las dimensiones que forman a su vez el SQT, para poder entender de forma más específica la naturaleza de la relación existente entre ambas variables consideradas respectivamente a nivel dimensional (Tabla 5) (Figuras 8 a 10). Los resultados obtenidos confirmaron una relación positiva y significativa entre la dimensión llusión (SQT) y la dimensión Consideración Individualizada (LT) ( $\beta=.28, p=.00)$. Sin embargo llusión (SQT) no se relacionó significativamente con ninguna de las restantes dimensiones de LT: Estimulación intelectual $(\beta=.03, p=.72)$, Inspiración $(\beta=.10, p=.46)$ y Carisma $(\beta=.05, p=.71)$. Por su parte, la dimensión Desgaste psíquico (SQT) no se relacionó significativamente con ninguna de las dimensiones de LT: Estimulación intelectual ( $\beta=-.02, p=.70)$, Inspiración $(\beta=-.12, \rho=.06)$, Consideración individualizada $(\beta=-.08, p=.23)$ y Carisma $(\beta=-.11, p=.10)$. La dimensión Indolencia (SQT) tampoco se relacionó significativamente con ninguna 
de las dimensiones de LT: Estimulación intelectual $(\beta=.06, p=.39)$, Inspiración $(\beta=-.02, \rho=.78)$, Consideración individualizada $(\beta=.02, p=.75)$ y Carisma $(\beta=-.02, p=.74)$. Dichos resultados permiten confirmar parcialmente la primera de las hipótesis planteadas en este apartado ( $\mathrm{H} 3 \mathrm{a})$. Sin embargo no fue posible confirmar ninguna de las dos siguientes $(\mathrm{H} 3 \mathrm{~b}$ y $\mathrm{H} 3 \mathrm{c}$ ) ya que en este caso no se encontraron influencias significativas a nivel dimensional entre las variables analizadas.

\section{Tabla 5}

\section{Resultados análisis dimensiones LT con dimensiones SQT (Regresión Stepwise)}

\begin{tabular}{|c|c|c|c|c|}
\hline & & Ilusión & & \\
\hline & Pasos & $\beta$ & $\mathrm{R}^{2}$ & $\mathrm{~F}$ \\
\hline \multirow{2}{*}{1} & Sexo & -.13 & \multirow{2}{*}{.02} & \multirow{2}{*}{$2.52^{\star}$} \\
\cline { 2 - 3 } & Edad & -.07 & & \\
\hline 2 & Cons. Individ. & $.28^{\star \star}$ & .08 & $7.72^{\star \star \star}$ \\
\hline
\end{tabular}

$$
{ }^{* * *} p \leq .001,{ }^{* *} p \leq .01,{ }^{*} p \leq .05
$$

Figura 8

Resultados Hipótesis 3a, modelo gráfico

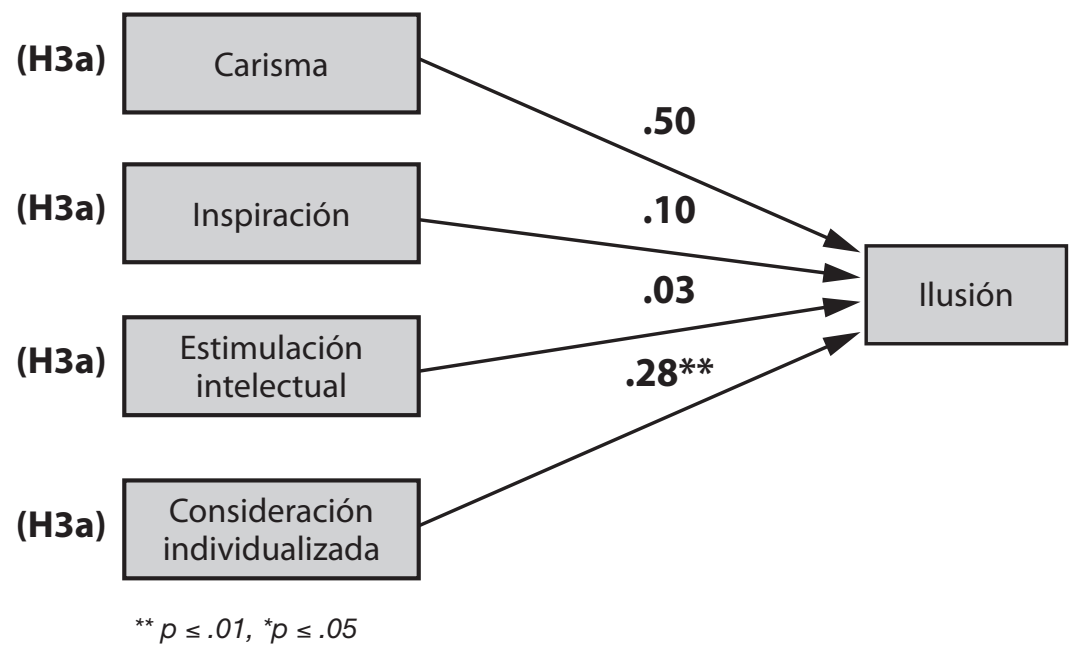




\section{Figura 9}

Resultados Hipótesis 3b, modelo gráfico

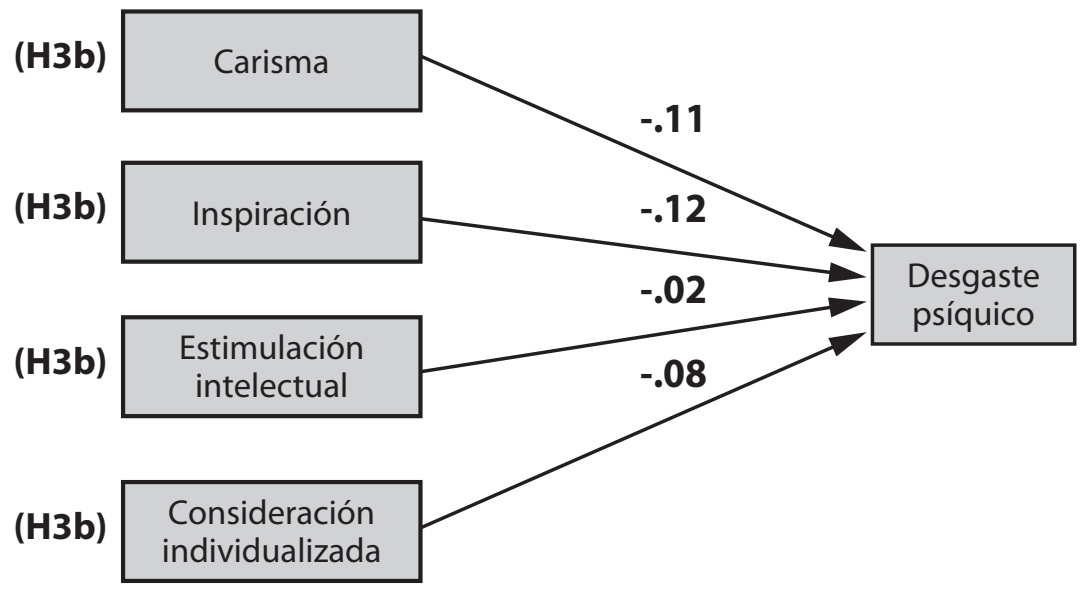

Figura 10

Resultados Hipótesis 3c, modelo gráfico

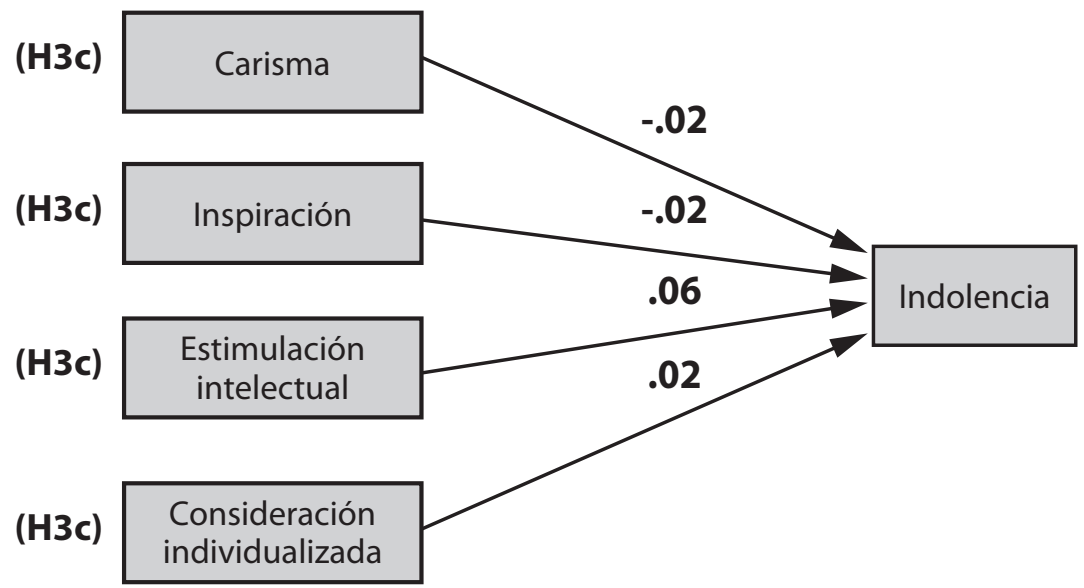




\section{DISCUSIÓN}

Los resultados obtenidos en el estudio muestran evidencias empíricas de que la aplicación del estilo de LT permite mejorar significativamente los niveles del SQT en la muestra de profesores de educación secundaria (ver Tabla 2 y Figura 6). Sin embargo, no se obtuvo evidencia empírica sobre las relaciones hipotetizadas entre LLF y SQT global (ver Tabla 2 y Figuras 1 y 6) ni entre LLF y las dimensiones componentes de SQT (ver Tabla 3), ya que en ambos casos LLF quedó excluida en las pruebas de regresión.

Los análisis realizados en ambas variables permiten avanzar en la especificidad de la relación planteada. En este sentido, el LT globalmente considerado permite incrementar la llusión en el trabajo de los empleados (ver Tabla 3). Por otra parte, considerando LT y SQT dimensión por dimensión, también se puede observar que la aplicación de conductas tendentes a la Consideración individualizada de los trabajadores mejora la llusión en el trabajo de los mismos (ver Tabla 5 y Figura 8). Aplicando dichas conductas, el líder consigue acercarse al empleado creando un contexto situacional basado en la atención particularizada, ya sea planteando una o varias reuniones periódicas en su oficina con el trabajador o trabajadora o bien únicamente a través de conversaciones de carácter más informal que pueden producirse directamente en el puesto de trabajo habitual del empleado.

Las interacciones descritas anteriormente se caracterizan en todo caso por la voluntad del líder de aproximarse a sus empleados desde un enfoque que trasciende las cuestiones meramente técnicas u operativas del puesto y se centra más bien en el componente humano que lo rodea, fomentando el crecimiento y desarrollo personal de cada uno de sus trabajadores.

Para conseguirlo el líder mantiene en todo momento una actitud basada en la escucha activa de las distintas necesidades, aspiraciones o expectativas individuales de cada empleado, tratando de ofrecerle su atención, apoyo y asesoramiento en las cuestiones planteadas y transmitiéndole la importancia de su contribución individual en el logro de los objetivos globales de la organización (Bass, 1997).

De este modo se consigue un doble objetivo: por una parte el líder alcanza una mayor comprensión de las preocupaciones 
intrínsecas de cada empleado, por otra parte los trabajadores se sienten escuchados y apoyados ya que perciben un liderazgo implicado y tendente a la resolución de problemas y a la búsqueda de alternativas eficaces a los mismos. Este contexto facilita la interacción líder-seguidores y permite el desarrollo de unas relaciones interpersonales basadas en un clima de mayor confianza.

Siguiendo la línea de estudios anteriores (Jennings et al., 2008; Mendoza y García, 2013) es posible afirmar que el apoyo prestado por el líder transformacional a sus empleados permite incrementar su llusión en el trabajo y disminuir significativamente los problemas asociados a la presencia de SQT en el entorno laboral.

Por otra parte, los resultados obtenidos también destacan el papel de la Inspiración (dimensión componente del LT) en la reducción de los niveles de SQT (ver Tabla 4 y Figura 7). Mediante esta conducta el líder persigue motivar a sus empleados planteándoles objetivos ambiciosos pero alcanzables, claros y definidos, lo cual constituye un reto y un estímulo para todos ellos; paralelamente les muestra una visión de futuro entusiasta y fortalece sus niveles de autoestima al transmitirles la idea de que serán capaces de superar cualquier tipo de obstáculo o dificultad que pueda surgir. Este tipo de actuaciones permiten el impulso de comportamientos y actitudes positivas entre los empleados junto al desarrollo de un fuerte sentido de equipo y misión colectivas.

En este sentido la Inspiración hace referencia a la capacidad que posee el líder para comunicar y hacer llegar su visión a sus trabajadores (Cuadrado y Molero, 2002). Estudios previos han destacado los efectos positivos que permite la aplicación del LT sobre los niveles de motivación de los empleados, dichos efectos fueron superiores a los logrados utilizando otros estilo de liderazgo distintos (Hetland y Sandal, 2003; Judge y Bono, 2000), lo cual permite hablar de una influencia predominante que resalta el papel del LT y le otorga una singularidad específica frente al resto (Bass, 2008). De este modo el líder transformacional consigue mayores niveles de implicación al mismo tiempo que estimula y motiva a sus empleados hacia la consecución de los objetivos globales de la organización (Godoy y Bresó, 2013). 


\section{CONCLUSIONES}

Como conclusión, los líderes que desarrollan un estilo de LT pueden contribuir a la prevención y reducción del SQT (García et al., 2007; Trógolo et al., 2013) y con ello al desarrollo del capital humano que constituye su entorno laboral. El desarrollo de conductas de Inspiración contribuye a la mejora de los niveles de SQT. Por su parte, la aplicación de conductas de Consideración individualizada incrementa la llusión en el trabajo de los empleados.

Respecto al LLF, a pesar de lo indicado sobre el mismo en el apartado de introducción, los resultados obtenidos en este estudio no permiten confirmar que su aplicación influya significativamente sobre el SQT global ni tampoco en ninguna de sus dimensiones consideradas.

Finalmente, el impulso de políticas de recursos humanos que permitan la formación de líderes en competencias transformacionales dentro del contexto educativo se antoja clave en la gestión de las nuevas relaciones laborales que caracterizan al mercado laboral actual, haciendo posible conseguir mayores niveles de salud ocupacional, eficacia y rendimiento organizacional.

\section{Limitaciones del estudio}

Una de las limitaciones del estudio es la referente al sesgo cognitivo, ya que los resultados pueden verse influidos por la motivación y sinceridad de los participantes en el momento de responder al cuestionario. No obstante el tamaño de la muestra tiene la suficiente amplitud como para contribuir a la reducción de dicho sesgo.

Por otra parte, la muestra de participantes en el estudio no fue balanceada en cuanto a su composición por sexo, ya que el porcentaje de mujeres fue mayor. Sin embargo las semejanzas observadas en este sentido en estudios previos realizados en el mismo colectivo (Collie et al., 2012; Rabasa et al., 2016) así como las similitudes en países limítrofes (más del 55\% de docentes mujeres en educación secundaria registradas en España en 2015) (UNESCO, 2017) reflejan el contexto ampliamente feminizado dentro de este sector. 
Finalmente cabe mencionar las limitaciones referentes a la transversalidad del estudio presentado.

\section{Recomendaciones}

Una vez comprobada la influencia positiva del líder transformacional sobre los procesos relacionados con el desarrollo del SQT dentro del colectivo docente cabría plantearse la implicación potencial de nuevos estilos de liderazgo emergentes en este tipo de procesos. En este sentido algunos autores (Walumbwa et al., 2008) hacen referencia al estilo de liderazgo auténtico, basado en un comportamiento caracterizado por el desarrollo de relaciones éticas, íntegras y transparentes hacia los seguidores y por el impulso de capacidades psicológicas positivas tales como la autoconfianza, la esperanza, el optimismo y la resiliencia.

Por otra parte, también se recomienda realizar estudios de carácter transocupacional y transnacional para evaluar la influencia de los estilos de LT y LLF en nuevos contextos sociolaborales y culturales, lo cual permitiría añadir un mayor grado de contraste a los resultados obtenidos en este estudio.

En lo referente al estudio de las relaciones entre las variables planteadas en este trabajo, también podría ser de interés la consideración de los potenciales efectos indirectos (procesos de mediación) o de interacción (procesos de moderación) que pueden desarrollarse, lo cual sería interesante de cara a ampliar la gama de relaciones causales de estudio dentro de esta materia.

Respecto a la aplicación de las técnicas y conductas transformacionales a nivel organizacional se plantea la necesidad de desarrollar programas de aprendizaje socio-emocional en centros educativos que permitan profundizar en el conocimiento, adquisición y perfeccionamiento competencial de los líderes actuales. En este sentido los servicios de prevención de riesgos laborales (a través de su modalidad propia o ajena) han comenzado a desarrollar en los últimos años una importante labor formativa obteniendo resultados muy positivos, tal como señalan varios estudios recientes (Bisquerra et al., 2015; Brackett et al., 2007; Cabello et al., 2010).

Por último, tal como se ha comentado en el apartado anterior, el carácter transversal del estudio plantea la necesidad de 
realizar análisis longitudinales que permitan complementar los resultados obtenidos.

\section{REFERENCIAS BIBLIOGRÁFICAS}

Antoniou, A. S., Polychroni, F. y Vlachakis, A. N. (2006). Gender and age differences in occupational stress and professional burnout between primary and high-school teachers in Greece. Journal of Managerial Psychology, 21, 682- 690.

Bass, B. M. (1997). Does the transactional/transformational leadership paradigm transcend organizational and national boundaries?. American Psychologist, 52, 130-139.

Bass, B. M. (2008) Handbook of Leadership: Theory, Research, and Application. New York: Free Press.

Bass, B. M. y Avolio, B. J. (1990). Transformational leadership development: Manual for the Multifactor Leadership Questionnaire. Palo Alto, CA: Consulting Psychologist Press.

Betoret, F. D. (2006). Stressors, self-efficacy, coping resources, and burnout among secondary school teachers in Spain. Educational Psychology, 26, 519-539.

Bisquerra, R., Pérez, J. C. y García, E. (2015). Inteligencia emocional en educación. Madrid: Síntesis.

Brackett, M. A., Alster, B., Wolfe, C., Katulak, N. y Fale, E. (2007). Creating an emotionally intelligent school district: A skillbased approach. En R. Bar-On, J. G. Maree y M. J. Elias (Eds.). Educating people to be emotionally intelligent (pp. 123-137). Londres: Praeger Publishers.

Cabello, R., Ruiz-Aranda, D. y Fernández-Berrocal, P. (2010). Docentes emocionalmente inteligentes. Revista Electrónica Interuniversitaria de Formación del Profesorado, 13(1), 41-49.

Camargo, G. K., Nesello, F., Geremias, H., Lopes, F., Durán, A., Eumann, A. y Maffei, S. (2015). Fatores associados a piores níveis na escala de Burnout em professores da educação básica. Cadernos Saúde Coletiva, 23(3), 268-275.

Carlotto, M. S. y Palazzo, L. S. (2006). Síndrome de burnout e fatores associados: um estudo epidemiológico com professores. Cadernos de Saúde Pública, 22, 1017-1026. 
Collie, R. J., Shapka, J. D. y Perry, N. E. (2012). School climate and social-emotional learning: Predicting teacher stress, job satisfaction, and efficacy. Journal of Educational Psychology, 104, 1189-1204.

Cuadrado, I. y Molero, F. (2002). Liderazgo transformacional y género: autoevaluaciones de directivos y directivas españoles. Revista de Psicología del Trabajo y de las Organizaciones, 18, 39-55.

De la Cruz, M. A. y Urdiales, M. C. (1996). Estrés del profesor universitario. Ansiedad y Estrés, 2, 261-282.

Doménech, F. y Gómez, A. (2010). Barriers perceived by teachers at work, coping strategies, self-efficacy and burnout. The Spanish Journal of Psychology, 13, 637-654.

European Agency for Safety and Health at Work (EU-OSHA) (2010). European Survey of Enterprises on New and Emerging Risks (ESENER). Luxembourg: Publications Office of the European Union. Recuperado de https://osha.europa.eu/es/ tools-and-publications/publications/reports/esener1_osh_ management/view

European Agency for Safety and Health at Work (EU-OSHA) (2015). Second European Survey of Enterprises on New and Emerging Risks (ESENER-2) (Summary). Luxembourg: Publications Office of the European Union. Recuperado de https:// osha.europa.eu/sites/default/files/publications/documents/ esener-ii-summary-en.PDF

Figueiredo-Ferraz, H., Gil-Monte, P. R. y Grau, E. (2009). Prevalencia del síndrome de quemarse por el trabajo (Burnout) en una muestra de maestros portugueses. Aletheia, 29, 6-15.

Figueiredo-Ferraz, H., Gil-Monte, P. R. y Grau-Alberola, E. (2013). Psychometric properties of the "Spanish Burnout Inventory" (SBI): Adaptation and validation in a Portuguese-speaking sample. European Review of Applied Psychology, 63, 33-40.

García, M., Rubio, P. y Bravo, L. (2007). Relación entre los factores de riesgo psicosocial y la resistencia al cambio organizacional en personal de seguridad de una empresa del sector económico terciario. Diversitas Perspectivas en Psicología, 3, 301-315.

Gastaldi, F. G. M., Pasta, T., Longobardi, C., Prino, L. E. y Quaglia, R. (2014). Measuring the influence of stress and burnout in 
teacher-child relationship. European Journal of Education and Psychology, 7, 17-28.

Gil-Monte, P. R. (2005). El síndrome de quemarse por el trabajo («burnout»). Una enfermedad laboral en la sociedad del bienestar. Madrid: Pirámide.

Gil-Monte, P. R. (2011). CESQT. Cuestionario para la evaluación del Síndrome de Quemarse por el Trabajo. Manual. Madrid: TEA Ediciones.

Gil-Monte, P. R., Carlotto, M. S., y Gonçalves, S. (2010). Validation of the Brazilian version of the "Spanish Burnout Inventory" in teachers. Revista de Saúde Pública, 44, 140-147.

Gil-Monte, P. R. y Figueiredo-Ferraz, H. (2013). Psychometric properties of the 'Spanish Burnout Inventory' among employees working with people with intellectual disability. Journal of Intellectual Disability Research, 57, 959-968.

Godoy, R. y Bresó, E. (2013). ¿Es el liderazgo transformacional determinante en la motivación intrínseca de los seguidores? Revista de Psicología del Trabajo y de las Organizaciones, 29(2), 59-64.

Hetland, H. y Sandal, G. M. (2003). Transformational leadership in Norway: Outcomes and personality correlates. European Journal of Work and Organizational Psychology, 12, 147-170.

Hetland, H., Sandal, G. M. y Johnsen, T. B. (2007). Burnout in the information technology sector: does leadership matter? European Journal of Work and Organizational Psychology, 16, 58-75.

Jennings, B., Disch, J. y Senn, L. (2008). Leadership. En R. G. Hughes (Ed.). Patient safety and quality: An evidence-based handbook for nurses (pp. 1-23). Rockville, MD: Agency for Healthcare Research and Quality.

Judge, A. T. y Bono, J. (2000). Five factor model of personality and transformational leadership. Journal of Applied Psychology, 85, 751-765.

Kanste, O., Kynga, H. y Nikkila, J. (2007). The relationship between multidimensional leadership and burnout among nursing staff. Journal of Nursing Management, 15, 731-739.

Kokkinos, C. M. (2007). Job stressors, personality and burnout in primary school teachers. British Journal of Educational Psychology, 77, 229-243. 
Liu, S. y Onwuegbuzie, A. J. (2012). Chinese teachers' work stress and their turnover intention. International Journal of Educational Research, 53, 160-170.

Longas, J., Chamarro, A., Riera, J. y Cladellas, R. (2012). La incidencia del contexto interno docente en la aparición del síndrome del quemado por el trabajo en profesionales de la enseñanza. Revista de Psicología del Trabajo y de las Organizaciones, 28(2), 107-118.

Martínez, J. P. (2015). Cómo se defiende el profesorado de secundaria del estrés: burnout y estrategias de afrontamiento. Journal of Work and Organizational Psychology, 31, 1-9.

Mendoza, I. A. y García, B. R. (2013). Liderazgo transformacional y desgaste profesional de personal docente de Escuelas Normales del Estado de México. Revista del Centro de Investigación. Universidad La Salle (México), 10(39), 57-79.

Mercado, A. y Gil-Monte, P.R. (2012). Características psicométricas del "Cuestionario para la evaluación del Síndrome de quemarse por el trabajo" en maestros mexicanos. Revista de Educación, 359, 260-273.

Mittal, R. (2015). Charismatic and transformational leadership styles: A cross-cultural perspective. International Journal of Business and Management, 10(3), 26-33.

Moriana, J. A. y Herruzo, J. (2004). Estrés y burnout en profesores. International Journal of Clinical and Health Psychology, 4, 597621.

Pacsi, A., Estrada, W., Pérez, A. y Cruz, P. (2014). Liderazgo laissez faire. Revista de Investigación Valor Agregado, 1(1), 67-72.

Pérez, J. (2010). ¿Es seguro el trabajo docente? Gestión práctica de riesgos laborales: Integración y desarrollo de la gestión de la prevención, 71, 18-23.

Rabasa B., Figueiredo-Ferraz H., Gil-Monte P.R. y M. LlorcaPellicer (2016). The Role of Guilt in the Relationship Between Teacher's Job Burnout Syndrome and the Inclination Toward Absenteeism. Revista de Psicodidactica, 21, 103-119.

Ratto, A., García, R. C., Silva, M. I. y González, M. C. (2015). El síndrome de quemarse por el trabajo (burnout syndrome) y factores psicosociales en docentes de primaria de la ciudad de Montevideo. Ciencias Psicológicas 9(2), 273 - 281. 
Richmond, V. P., Wrench, J. S. y Gorham, J. (2009). Communication, affect and learning in the classroom (3rd. ed.). Edina, MN: Burgess Publishing.

Rome, K.P. (2000). The palliative effect of leadership agents on reactions to workplace stressors. Dissertation Abstracts International, 60(11-A). US: University Microfilms International.

Salanova, M. y Llorens, S. (2008). Estado actual y retos futuros en el estudio del burnout. Papeles del Psicólogo, 29(1), 59-67.

Skaalvik, E. M. y Skaalvik, S. (2007). Dimensions of teacher self-efficacy and relations with strain factors, perceived collective teacher efficacy, and teacher burnout. Journal of Educational Psychology, 99, 611- 625.

Trógolo, M. A., Pereyra, A. P. y Sponton, C. (2013). Impacto de diferentes estilos de liderazgo sobre el Engagement y Burnout: Evidencia en una muestra de trabajadores argentinos. Ciencia y Trabajo, 15(48), 152-157.

Unda, S. (2010). Estudio de prevalencia del Síndrome de Quemarse por el Trabajo (SQT) y su Asociación con Sobrecarga y Autoeficacia en Maestros de Primaria de la Ciudad de México. Ciencia y Trabajo, 12(35), 257-262.

Unda, S., Sandoval, J. y Gil-Monte, P. R. (2008). Prevalencia del Síndrome de Quemarse por el Trabajo (SQT) (burnout) en maestros mexicanos. Información Psicología, 91, 53-63.

UNESCO Institute for Statistics (UIS.Stat) (2017). Percentage of female teachers by teaching level of education. Recuperado de http://data.uis.unesco.org/Index.aspx

Walumbwa, F. O., Avolio, B. J., Gardner, W. L., Wernsing, T.S. y Peterson, S.J. (2008). Authentic leadership: Development and validation of a theory-based measure. Journal of Management, 34(1), 89-126.

Wiegel, C., Sattler, S., Göritz, A. S. y Diewald, M. (2016). Work-related stress and cognitive enhancement among university teachers. Anxiety Stress Coping, 29(1), 100-117. 